\title{
面向可重构扫描网络的锁定隔离安全结构
}

\author{
刘军1)，王亭亭1)，任福继1,2) \\ ${ }^{1)}$ (合肥工业大学计算机与信息学院人工智能学院 合肥 230601) \\ 2) (Graduate School of Advanced Technology \& Science, University of Tokushima Tokushima 7708502) \\ (liujun@hfut.edu.cn)
}

\begin{abstract}
摘 要: 为使可重构扫描网络免受未经授权的访问、恶意仪器对传输数据的鼌改和嗅探 3 种安全攻击的影响, 提出 了锁定隔离安全结构. 该结构首先把彼此不具有安全威胁的仪器分成一组, 通过控制隔离信号实现组与组之间的单 独访问，以防止恶意仪器对传输数据的害改和嗅探. 然后通过使用锁段插入位保护关键的仪器，只有扫描网络中处 于特定位置的多个键值被设置成特定值 $(0,1$ 序列)时锁段插人位才能打开, 能加大未经授权访问的难度. 此外, 为解 决仪器分组多导致硬件开销大和布线困难的问题, 提出了仪器分组算法, 根据仪器间的安全关系构建无向图, 然后 对无向图求极大独立集, 能有效地减少仪器分组数. 在 ITC 02 基准电路上的实验结果表明, 与国际上同类方法相比, 所提的安全结构打开锁段插人位所需要的时间增大了 7 倍，在面积、功耗和布线上的平均减少百分比分别为 $3.81 \%$, $9.02 \%$ 和 $4.55 \%$.
\end{abstract}

关键词：可重构扫描网络; 安全攻击; 隔离信号; 锁段插人位

中图法分类号: TP391.41 DOI: 10.3724/SP.J.1089.2021.18447

\section{Lock Isolation Security Architecture for Reconfigurable Scanning Networks}

\author{
Liu Jun ${ }^{1)}$, Wang Tingting ${ }^{1)}$, and Ren Fuji, ${ }^{1,2)}$ \\ 1) (School of Computer Science and Information Engineering, School of Artificial Intelligence, Hefei University of Technology, Hefei 230601) \\ 2) (Graduate School of Advanced Technology \& Science, University of Tokushima, Tokushima 7708502)
}

\begin{abstract}
To protect the reconfigurable scanning network from the influence of unauthorized access, tampering of transmitted data by malicious instruments and sniffing, a lock isolation security structure is proposed. Firstly, the presented structure divides the instruments without security threat to each other into one group, and the groups can be accessed one by one via the isolated signals, which can prevent malicious instruments tampering and sniffing transmitted data. Secondly, the key instruments are protected by the lock segment insertion bit. The lock segment insertion bit can only be opened when multiple key values at specific positions are set to specific values ( 0,1 sequence), which can increase the difficulty of unauthorized access. Besides, to reduce the hardware overhead, and the difficulty of place and route caused by excessive instrument groups, instrument grouping algorithm is proposed. The proposed algorithm firstly constructs an undirected graph based on the security relationship among instruments. Then the maximal independent sets of the undirected are obtained. In this way, the number of instrument groups is reduced greatly. Compared to other similar methodologies, the experimental results conducted on ITC 02 benchmark circuits show that the time consumption for opening the locking segment insertion bit of the proposed security structure increased by 7 times, and the average percentage reduction of area, power consumption and wire length is $3.81 \%, 9.02 \%$ and $4.55 \%$, respectively.
\end{abstract}

收稿日期: 2020-06-23; 修回日期: 2020-12-07. 基金项目：国家自然科学基金(U1613217). 刘军(1978一), 男, 博士，副教授，硕 士生导师, CCF 会员, 主要研究方向为数字系统设计自动化集成电路测试、嵌人式系统; 王亭亭(1996一), 女, 硕士研究生, 主要研究 方向为高可靠性嵌人式系统; 任福继(1959-), 男, 博士, 教授, 博士生导师, 主要研究方向为智能机器人. 
Key words: reconfigurable scan network; security attacks; isolated signal; lock segment insert bit

随着集成电路集成度和复杂性的增加，单个 芯片上集成的功能模块越来越多，如用于可测试 性设计的部件、传感器、监视器和调试控制器等, 大量的这种功能模块需要被测试访问，那么灵活 的访问机制将是必要的.

IEEE P1687 标准 ${ }^{[1]}$ 也被称为内部联合测试行 动组(internal joint test action group, IJTAG), 使用 段插人位(segment insert bit, SIB)构建可重构扫描 网络，通过向 SIB 移人配置数据改变扫描链.

虽然在可重构扫描网络中可以灵活地配置扫 描路径，但是也存在着一些安全问题 ${ }^{[2-3]}$. 第 1 种 安全问题称为未经授权的访问. 攻击者把配置数 据移人 SIB, 通过比较移人数据和移出数据的长度 判断是否打开了新的仪器，通过不断尝试得到整 个网络的结构, 便可以通过移位操作得到仪器中 的重要数据. 第 2 种安全问题称为数据传输攻击, 是指芯片上的仪器本身是恶意的，可能会改变当 前扫描网络中 SIB 的配置数据, 进而打开目前不需 要访问的仪器或强制关闭正在访问的仪器. 此外, 恶意仪器还可能改变扫描网络的输人数据或输出 数据, 进而造成测试失败等后果. 第 3 种安全问题 称为数据嗅探, 恶意仪器可能会跟踪流经自身的 数据并对一些重要数据进行推断来获得一些重要 信息，如与 SIB 密钥相关的数据等.

研究者对可重构扫描网络的安全性进行了大 量的研究, 这些研究工作可以分为 2 类, 一类是增 大未经授权的访问难度 ${ }^{[4-12]}$; 另一类是防止恶意仪 器对传输数据的害改和嗅探攻击 ${ }^{[13-16]}$. 文献[4-5] 通过使用多个分布式密钥位增大未经授权用户对 重要仪器访问的难度. 文献[6-7]分别通过线性反 馈移位寄存器和物理不可克隆函数生成密钥, 增 加了解锁 SIB 的难度. 为了进一步增加攻击者访问 难度, 文献[8]通过密码流保护输人输出的数据, 同时将一些额外的扫描链插人可重构扫描网络中 混淆 IJTAG 网络结构，使攻击者更难推测出 IJTAG 网络结构. 文献[9-11]在测试访问端口和扫描网络 之间放置序列过滤器, 如果移人的数据涉及受保 护仪器的访问, 过滤器就通过控制更新信号阻止 所有仪器的更新操作. 文献[9-11] 虽然可以限制未 经授权的访问, 但是不能灵活地访问单个仪器. 为 实现对单个仪器的灵活访问, 文献[12]提出了一种 基于质询-响应认证协议的可重构扫描网络访问管 理方法，使用扩展的 SIB 控制单个设备访问，只有
当 SIB 状态位为 1 且经过认证该 SIB 保护的仪器 能访问时, 此 SIB 才能进行更新操作. 以上工作加 大了未经授权用户的访问难度，但不能防止恶意 仪器对传输数据的宫改和嗅探. 文献[13]使用数据 选择器对恶意仪器进行旁路, 以保护网络免受恶 意仪器对传输数据的宫改攻击和嗅探攻击. 文献 [14]为每一个仪器增加一个额外的扫描链, 此扫描 链称为阴影扫描链, 其长度与仪器的原扫描链长 度相等. 在仪器测试期间, 比较原扫描链和阴影扫 描链的数据, 若两者数据不同, 则流经的恶意仪器 对数据进行了窝改. 文献[15]在不改变扫描网络结 构的前提下, 通过对恶意仪器进行隔离, 可防止恶 意仪器对数据的修改和嗅探. 为了不使用额外的 数据选择器和扫描链, 文献[16]通过遍历可重构扫 描网络的结构识别不安全的路径, 然后使用重布线 消除每一个不安全路径，有效地降低了硬件开销.

通过上述工作，能增大 IJTAG 网络中未经授 权用户的访问难度 ${ }^{[4-12]}$, 能使 IJTAG 网络免受恶意 仪器对传输数据的嗅探或窝改 ${ }^{[13-16]}$, 但是都未能 把 3 种安全问题结合起来考虑. 本文将 3 种安全问 题相结合, 提出了锁定隔离安全结构, 不仅能防止 恶意仪器对传输数据的窝改和嗅探, 同时可以加 大未经授权访问的难度. 该结构根据仪器之间的 安全关系对仪器进行分组, 通过控制隔离信号实 现组与组之间的单独访问. 此外, 通过锁段插人位 (lock segment insert bit, LSIB) 隐藏关键仪器, 以确 保此仪器只能被授权的用户访问.

\section{1 锁定隔离安全结构}

锁定隔离安全结构根据仪器之间的安全关系 把仪器分成若干组, 一个组内的所有仪器彼此都 不存在安全威胁, 即在同一分组内, 每个仪器的数 据都可流经组内其他仪器而不用担心数据会被修 改和嗅探. 2 个仪器之间存在安全威胁是指若流经 仪器 $A(B)$ 的数据由于安全问题不能流经仪器 $B(A)$, 就称仪器 $A$ 与仪器 $B$ 存在安全威胁.

仪器组之间的单独访问通过译码器输人端的 隔离信号进行控制, 若结构中有 $b$ 个仪器组, 则译 码器的输人端需要 $\left\lceil 1 b^{b}\right\rceil$ 个隔离信号, 每个仪器组 旁路与否需要一个多路选择器和一个与门来实现. 图 1 所示为此结构的一个示例, 包括 4 个仪器组 


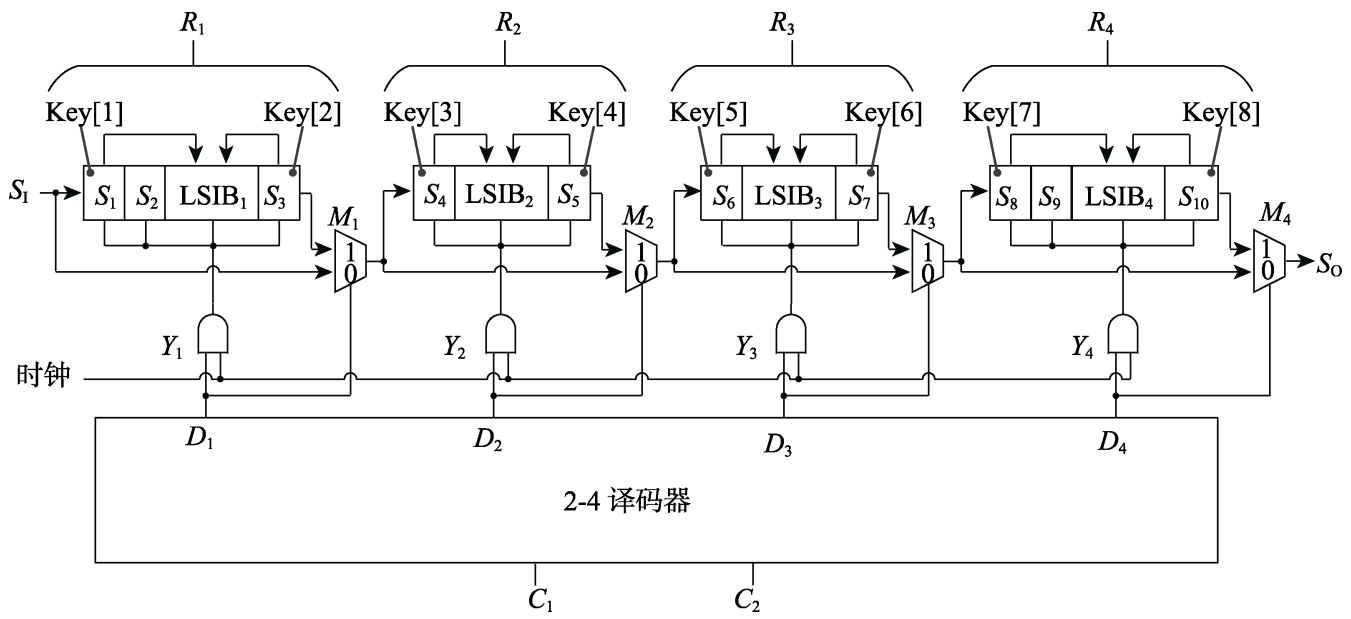

图 1 锁定隔离安全结构

$R_{1}, R_{2}, R_{3}$ 和 $R_{4}, C_{1}$ 和 $C_{2}$ 是控制仪器组单独访 问的隔离信号. 2 个隔离信号产生 4 种不同状态组 合分别控制 4 个仪器组的访问.

隔离信号 $C_{1}$ 和 $C_{2}$ 不同状态组合在控制数据 移动阶段的情况如下. 当 $C_{1}=0, C_{2}=0$ 时, 输出端 $D_{1}$ 为高电平, $D_{2}, D_{3}$ 和 $D_{4}$ 为低电平. 此时与门 $Y_{1}$ 的输出为时钟信号, 仪器组 $R_{1}$ 便可进行数据的移 位操作, 数据选择器 $M_{1}$ 把仪器组 $R_{1}$ 包含在扫描 路径中. 与门 $Y_{2}, Y_{3}$ 和 $Y_{4}$ 的输出全为 0 , 相应的仪 器组 $R_{2}, R_{3}$ 和 $R_{4}$ 不能进行数据移位, 数据选择 器 $M_{2}, M_{3}$ 和 $M_{4}$ 分别把仪器组 $R_{2}, R_{3}$ 和 $R_{4}$ 进 行旁路. 此状态是单独访问仪器组 $R_{1}$ 的情况.

同理, 当 $C_{1}=0, C_{2}=1$ 时, 译码器的输出端 $D_{2}$ 输出为高电平, $D_{1}, D_{3}$ 和 $D_{4}$ 输出为低电平, 此时 是单独访问仪器组 $R_{2} ; C_{1}=1, C_{2}=0$ 时, 能单独 访问 $R_{3} ; C_{1}=1, C_{2}=1$ 时, 则能单独访问 $R_{4}$.

可见，在图 1 所示的锁定隔离安全结构中，流 经某个仪器组的数据并没有通过其他仪器组, 又 因为组内所有仪器都不存在安全威胁, 每个仪器 的数据都不会受到组内其他仪器的攻击. 这样便 能保证整个结构中的数据在传输中不会受到恶意 仪器的窝改和嗅探攻击.

此外，在锁定隔离安全结构中，对于安全要求 比较高的仪器(如只提供给特定用户访问的仪器) 采用 LSIB 控制访问. 与普通 SIB 相比，控制 LSIB 更新操作的信号除 UpdateEn 信号之外，还有若干 个密钥位. 只有当 UpdateEn 信号为 1 且所有的密 钥位都被设置成特定值(1 或 0)时, LSIB 才能实现 更新操作. LSIB 的结构如图 2 所示, 此 LSIB 包含 $\operatorname{Key}[1], \operatorname{Key}[2], \cdots, \operatorname{Key}[k]$ 共 $k$ 个密钥位，密钥位
可以是 IEEE P1687 网络中本就存在的 SIB, 也可 以是新添加的不连接任何仪器的 SIB. 例如, 在图 1 锁定隔离安全结构中, $S_{1}, S_{2}, \cdots, S_{10}$ 是连接嵌人 式仪器的 SIB, 4 个仪器组都含有 1 个 LSIB, 如 $\mathrm{LSIB}_{1}$ 的密钥位由 $S_{1}$ 和 $S_{3}$ 组成, 只有 $S_{1}$ 和 $S_{3}$ 都被 移人特定值时 $\mathrm{LSIB}_{1}$ 才能进行更新操作.

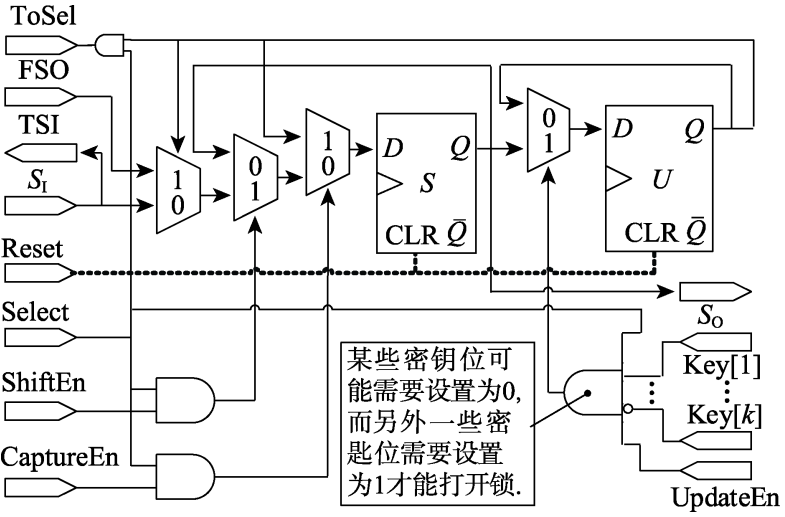

图 2 LSIB 结构

使用 LSIB 可以增大未经授权访问难度，通过 译码器输人端的隔离信号控制访问仪器组也能增 大未经授权访问难度. 例如, 在图 1 结构中需要单 独访问仪器组 $R_{4}$, 此时译码器的隔离信号 $C_{1}$ 和 $C_{2}$ 的状态组合有 $00,01,10$ 和 11 共 4 种可能, 2 个 隔离信号的随机状态组合能成功访问仪器组 $R_{4}$ 的 概率为 $1 / 4$. 如果在锁定隔离安全结构中有 $b$ 个仪 器组, 就需要 $\left\lceil\mathrm{lb}^{b}\right\rceil$ 个隔离信号. 隔离信号的随机 一种状态组合能打开某个特定仪器组的概率为 $1 / b$. 攻击者试图打开 LSIB 保护的仪器时, 首先 需要随机猜测隔离信号的状态组合, 若组合正确, 则能成功打开仪器组; 接下来还要继续猜测 LSIB 
密钥的位置, 密钥的个数以及密钥的取值才有可 能打开受保护的仪器, 从而获得重要数据. 隔离信 号和 LSIB 的双重保护增大了未经授权用户访问的 难度.

锁定隔离安全结构的提出主要是为了解决可 重构扫描网络中存在的 3 种安全问题. 通过在可重 构扫描网络中加人 LSIB 和对仪器进行分组隔离相 结合的方法, 既能加大未经授权的访问难度，也能 防止恶意仪器对数据的鼌改和嗅探.

\section{2 优化仪器分组}

仪器组的单独访问由隔离信号控制, 仪器分 组过多将导致硬件开销大和布线困难. 因为仪器 的用途不同对安全的要求也不同，所以可以根据 仪器的不同安全性减少分组数. 例如, 用于生成关 键密钥的加密仪器, 由于较高的安全要求, 不应让 其数据流经其他任何仪器, 因为它可能被操纵或 嗅探信息. 类似地, 跟踪缓冲区可能包含一些重要 信息, 应该避免其数据流经恶意仪器, 但是其数据 可以流经其他安全仪器. 同时, 部分仪器不需要任 何安全性, 其数据可以通过任何仪器, 没有安全风 险. 这些不同的安全关系可用于减少仪器分组数. 与文献[15]相比, 本文的仪器分组算法利用求无向

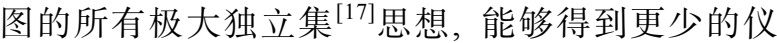
器分组数.

本文把仪器之间的安全关系建模成无向图, 通过求无向图的所有极大独立集减少仪器分组数. 每个极大独立集包含的所有仪器作为一个分组, 在原无向图上去掉独立集包含的仪器顶点, 对剩 下的仪器顶点再进行分组. 通过广度优先建树的 方法把求出的所有极大独立集作为树的节点来存 储. 建树过程中首先到达叶子节点(完成所有仪器 分组)的那条路径上所有的极大独立集就是仪器的 最少分组情况, 树的最小层次数就是仪器分组数.

算法 1. 仪器分组算法.

输人. $K$ 个仪器及仪器之间的安全关系.

输出. 仪器分组数 $M$ 和 $M$ 个集合, 每个集合 中包含的所有仪器构成一个仪器组.

Step1. 建立只有根节点 $A_{0}$ 的树 $T$, 节点 $A_{0}$ 对应的 层数为 0 , 建立空队列 $Q$.

Step2. 把仪器和仪器之间的安全关系建模成一个 无向图, 无向图用邻接矩阵 $\boldsymbol{G}_{i}$ 表示, 同时把 $\boldsymbol{G}_{0}$ 加人队 列 $Q$.

Step3．队列 $Q$ 非空时循环执行此步骤:
Step3.1. 出队列得到邻接矩阵 $\boldsymbol{G}_{i}$;

Step3.2. 若 $\boldsymbol{G}_{i}$ 不为空, 执行极大独立集算法得到 $\boldsymbol{G}_{i}$ 的所有 $N$ 个极大独立集 $A_{L+1}, A_{L+2}, \cdots A_{L+N}$, 同时把它们 作为树节点 $A$ 的 $N$ 个孩子节点并设置所在层数, 从矩 阵 $\boldsymbol{G}_{i}$ 中去除独立集 $A_{j}(j=1,2, \cdots, N)$ 所包含顶点的行和 列, 得到新的邻接矩阵 $\boldsymbol{G}_{j}$, 并将 $\boldsymbol{G}_{j}$ 加人 $Q$ 队列;

Step3.3. 若 $\boldsymbol{G}_{i}$ 为空, 此时完成所有仪器的分组且 分组数最少, 得到分组数和分组集合.

其中, 极大独立集算法是通过图分解方法求 出邻接矩阵 $\boldsymbol{G}$ 的所有极大独立集. 对于邻接矩阵 $\boldsymbol{G}, \boldsymbol{G}^{*} V$ 表示 $\boldsymbol{G}$ 去掉顶点 $V$ 和所有与 $V$ 相连的顶点 和边后得到的邻接矩阵, $\boldsymbol{G}-V$ 表示 $\boldsymbol{G}$ 去掉顶点 $V$ 和 所有与 $V$ 相连的边后得到的邻接矩阵. 设 $V$ 为邻 接矩阵 $\boldsymbol{G}$ 的任一顶点, $\boldsymbol{G}$ 的所有极大独立集构成的 集合表示为

$S(G)=V^{*} S(G * V)+\{S \mid S \in S(G-V), S \cap N(V) \neq \varnothing\}$. 其中， $N(V)$ 表示所有与顶点 $V$ 相连的顶点.

算法 2. 极大独立集算法.

输人. 邻接矩阵 $\boldsymbol{G}$.

输出. $G$ 的所有极大独立集.

Step1. 如果 $\boldsymbol{G}$ 为空矩阵, 返回求得的极大独立集 合; 若矩阵 $\boldsymbol{G}$ 的阶数为 2 , 把 2 个顶点分别加人求得的 极大独立集，返回新的极大独立集合.

Step2. 选取邻接矩阵 $\boldsymbol{G}$ 中度数最大的顶点 $V$, 对 $\boldsymbol{G}$ 做变换分别得到 $G^{*} V$ 矩阵和 $\boldsymbol{G}-V$ 矩阵.

Step3. 如果 $G^{*} V$ 矩阵中存在元素全为 0 的行(列), 找出所有这样的行(列), 设这些行(列)所对应的图的顶 点为 $U_{1}, U_{2}, \cdots, U_{m}$, 以新矩阵 $G^{*} V-U_{1}-U_{2}-\cdots-U_{m}$ 为参 数递归执行此算法. 如果矩阵 $G^{*} V$ 中不存在元素全为 0 的行(列), 则以矩阵 $G^{*} V$ 为参数递归执行此算法.

Step4. 如果矩阵 $\boldsymbol{G}-V$ 中存在元素全为 0 的行(列), 找出所有这样的行(列), 设这些行(列)所对应的图的顶 点为 $W_{1}, W_{2}, \cdots, W_{f}$, 以新矩阵 $\boldsymbol{G}-V-W_{1}-W_{2}-\cdots-W_{f}$ 为参 数递归执行此算法. 如果矩阵 $\boldsymbol{G}-V$ 中不存在元素全为 0 的行(列), 则以矩阵 $\boldsymbol{G}-V$ 为参数递归执行此算法.

\section{3 实验和评估}

\section{1 总体安全性评估}

本文方法与文献[4-16]在可重构扫描网络安全 方面进行比较, 结果如表 1 所示. 可重构扫描网络 安全方面存在的攻击类型共有 3 种, 分别是未经授 权访问攻击、数据宫改攻击和数据嗅探攻击. 由表 1 可以看出, 文献[4-12]能加大未经授权的访问难 度, 文献[13-16]能预防数据宫改攻击和数据嗅探 攻击. 本文提出的锁定隔离安全结构在加大未经 
授权访问难度的同时也能防止恶意仪器对数据的 菴改和嗅探攻击.

表 1 安全性分析

\begin{tabular}{lccc}
\hline \multicolumn{1}{c}{ 攻击类型 } & 文献[4-12] & 文献[13-16] & 本文 \\
\hline 未经授权访问 & $\sqrt{ }$ & $\times$ & $\sqrt{ }$ \\
数据审改 & $\times$ & $\sqrt{ }$ & $\sqrt{ }$ \\
数据嗅探 & $\times$ & $\sqrt{ }$ & $\sqrt{ }$ \\
\hline
\end{tabular}

\section{2 未经授权访问安全性评估}

为了评估本文提出的结构对于未经授权访问 的安全性，考虑一个简单的单层可重构扫描网络 结构, 其包含多个仪器, 每个仪器连接一个 SIB, 其中存在一个安全性较高的仪器通过连接 LSIB 进 行安全保护. 当 LSIB 关闭时, 整个扫描路径的长 度为 $n$, 密钥位个数为 $k$, 仪器组个数为 $b$. 如果 锁定隔离安全结构中有 $b$ 个仪器组, 那么以随机 $\left\lceil\mathrm{lb}^{b}\right\rceil$ 个隔离信号的状态组合成功打开某一个特定 仪器组的概率为 $1 / b$. 此结构中若想访问 LSIB 保 护的仪器, 首先要对 $\left[\mathrm{lb}^{b}\right\rceil$ 个隔离信号(译码器的输 人端)状态进行组合尝试, 若经过多次尝试成功打 开仪器组, 还需要正确移人与 LSIB 相关联的 $k$ 个 密钥位的值, 每次尝试 $k$ 个密钥的值都需要移人 $n$ 位(整个扫描路径的长度). 表 2 展示了在时钟速度 为 $10 \mathrm{MHz}$ 时, 锁定隔离安全结构解锁 LSIB 与文 献[4]所要花费时间的比较.

表 2 解锁 LSIB 所需时间比较

\begin{tabular}{rrrccc}
\hline \multirow{2}{*}{$b$} & \multirow{2}{*}{$k$} & \multirow{2}{*}{$n$} & \multicolumn{2}{c}{ 所需时间 $/ \mathrm{d}$} & 增加时 \\
\cline { 4 - 5 } & & & 本文 & 文献 $[4]$ & 间倍数 \\
\hline 4 & 8 & 640 & $1.59 \times 10^{-6}$ & $3.97 \times 10^{-7}$ & 3 \\
5 & 16 & 1280 & $9.95 \times 10^{-4}$ & $1.99 \times 10^{-4}$ & 4 \\
6 & 32 & 2560 & $1.55 \times 10^{2}$ & $2.58 \times 10^{1}$ & 5 \\
7 & 48 & 5120 & $2.35 \times 10^{7}$ & $3.36 \times 10^{6}$ & 6 \\
8 & 64 & 10240 & $3.51 \times 10^{12}$ & $4.39 \times 10^{11}$ & 7 \\
9 & 80 & 20480 & $5.17 \times 10^{17}$ & $5.74 \times 10^{16}$ & 8 \\
10 & 96 & 40960 & $7.52 \times 10^{22}$ & $7.52 \times 10^{21}$ & 9 \\
\hline
\end{tabular}

由表 2 可以看出, 对于较少的隔离信号和密钥 位以及较小的扫描路径长度，如 $b=4, k=8$, $n=640$, 此结构打开特定仪器所需的时间小于 $10 \mathrm{~s}$. 对于 $b=6, k=32, n=2560$, 则需要约 155 天的 时间. 而当 $b=8, k=64, n=10240$ 时，所需时间超 过了 96 亿年. 相对于文献[4], 本文结构极大地增 加了未经授权访问的难度，打开一个 LSIB 所需时 间平均是文献[4]的 7 倍.

\section{3 隔离信号优化比较}

本文使用 ITC 02 基准电路 ${ }^{[18]}$ 对提出的仪器分 组算法进行评估，并与文献[15]进行比较. 在实验 中, 每个基准电路包含多个模块, 每个模块有多条 内部扫描链, 本文将每个模块内的每条扫描链都 视为一个仪器, 结果如表 3 所示.

表 3 仪器分组算法比较

\begin{tabular}{lrrrrrr}
\hline \multirow{2}{*}{ 电路名称 } & \multirow{2}{*}{ 仪器数量 } & \multicolumn{2}{c}{ 仪器分组数 } & & \multicolumn{2}{c}{ 隔离信号数 } \\
\cline { 3 - 4 } d281 & & 文献[15] & 本文 & & 文献[15] & 本文 \\
\hline p34392 & 34 & 11 & 9 & & 11 & 4 \\
d695 & 63 & 17 & 14 & & 17 & 4 \\
h953 & 137 & 26 & 21 & 26 & 5 \\
p22810 & 28 & 9 & 7 & 9 & 3 \\
a586710 & 196 & 35 & 28 & 35 & 5 \\
\hline
\end{tabular}

表 3 给出了在相同仪器和相同安全威胁的情 况下, 文献[15]与本文提出的仪器分组算法分别求 出仪器分组数和相应需要的隔离信号个数的比较. 可以看出，在相同的实验条件下，本文提出的仪器 分组算法求出的仪器分组数个数明显少于文献 [15]. 由于文献[15]中每一个仪器组都需要一个隔 离信号进行控制, 所以仪器分组数和隔离信号数 相等，而本文结构隔离信号连接在译码器的输人 端，隔离信号的不同状态组合控制各仪器组的访 问，所以需要更少的隔离信号.

\section{4 锁定隔离安全结构面积功耗布线分析}

本文使用 ITC 02 基准电路, 各电路元器件使 用 UMC $0.18 \mu \mathrm{m}$ 工艺对本文的锁定隔离安全结构 在面积、功耗和布线上进行评估，并与文献[15]进 行比较，结果如表 4 所示.

表 4 在面积以及功耗的比较上考虑了是否采 用译码器的 2 种结果. 实验数据的百分比表示本文

表 4 比文献[15]在面积、功耗和布线上减少的百分比 $\%$

\begin{tabular}{|c|c|c|c|c|c|}
\hline \multirow{2}{*}{ 电路名称 } & \multicolumn{2}{|c|}{ 未使用译码器 } & \multicolumn{2}{|c|}{ 使用译码器 } & \multirow{2}{*}{$\begin{array}{l}\text { 布线 } \\
\text { 长度 }\end{array}$} \\
\hline & 面积 & 功耗 & 面积 & 功耗 & \\
\hline $\mathrm{d} 281$ & 10.91 & 17.11 & 2.74 & 8.55 & 5.96 \\
\hline P34392 & 11.76 & 17.05 & 6.48 & 11.51 & -1.62 \\
\hline d695 & 13.85 & 18.97 & 6.93 & 11.02 & 6.47 \\
\hline h953 & 14.44 & 20.82 & 4.46 & 10.36 & -2.34 \\
\hline P22810 & 15.71 & 19.83 & 10.58 & 13.92 & 10.01 \\
\hline a586710 & 5.00 & 14.43 & -8.31 & -1.27 & 8.80 \\
\hline 平均百分比 & 11.95 & 18.04 & 3.81 & 9.02 & 4.55 \\
\hline
\end{tabular}


的面积或功耗相较于文献[15]减少的百分比，个别 负数表示比文献[15]有增多. 结果表明，与文献 [15]相比，本文所提的锁定隔离安全结构若不采用 译码器, 在面积和功耗上平均减少的百分比分别 为 $11.95 \%$ 和 $18.04 \%$; 若采用译码器减少隔离信号 的个数, 由于加上译码器的面积和功耗, 此结构在 面积和功耗上平均减少的百分比分别为 $3.81 \%$ 和 $9.02 \%$; 在布线长度上，本文算法比文献[15]平均 减少 $4.55 \%$.

实验数据显示, 从包含 16 个仪器的 a586710 电路到包含 196 个仪器的 p22810 电路, 相应的面 积以及功耗平均减少的百分比为 $3.81 \%$ 和 $9.02 \%$, 并未导致芯片面积和功耗的大幅增加.

\section{5 锁定隔离安全结构在 AES 加密电路中的 应用}

在高级加密标准 (advanced encryption standard, AES)加密电路中，主要包含 4 个步骤：字节 替代、行移位、列混合和轮密相加. 例如, AES-128 中密钥的长度为 128 位, 加密轮数为 10 轮. 1 9 轮 包括字节替换、行移位、列混合与轮密相加运算, 末轮变换与普通轮变换基本相同, 只是没有列混 合运算. 攻击者可以通过攻击手段得到字节替换、 行移位、列混合和轮密相加运算后中间变量的值, 进而推测密钥.

针对 AES 加密电路中存在的上述安全问题, 可以运用本文提出的锁定隔离安全结构加大破解 AES 密钥的难度. 在 AES 加密电路中加人多个键 值，通过键值位不同的取值组合生成掩码，在每种 加密运算过程中添加掩码，将中间值隐藏; 然后在 轮变换的输出端消除随机掩码, 攻击者便不能通 过加密运算过程中的中间值推测密钥.

\section{4 结 语}

本文提出了一种能解决可重构扫描网络中存 在的 3 种安全攻击的锁定隔离安全结构. 该结构可 以在数据移人和移出阶段把恶意仪器隔离, 阻止 数据通过恶意仪器从而防止数据宫改攻击和数据 嗅探攻击; 通过在扫描网络中使用 LSIB 保护安全 性较高的仪器，只有在多个密钥位都输人特定的 值时才能访问 LSIB 所保护的仪器，增大了未经授 权的访问难度，同时隔离信号的不同状态组合也 加大了未经授权访问难度. 此外, 本文提出的仪器 分组算法可以根据仪器间的安全关系求出更少的 仪器分组数, 解决了仪器分组过多导致的面积开
销大和布线困难的问题. 因此，本文提出的方案非 常适合 IEEE P1687 标准下的现代复杂系统芯片设 计，可以有效防止 3 种恶意攻击.

\section{参考文献(References):}

[1] IEEE. IEEE Standard for Access and Control of Instrumentation Embedded within a Semiconductor Device: 1687-2014[S]. Los Alamitos: IEEE Computer Society Press, 2014

[2] Dworak J, Crouch A. A call to action: securing IEEE 1687 and the need for an IEEE test security standard[C] //Proceedings of the 33rd IEEE VLSI Test Symposium. Los Alamitos: IEEE Computer Society Press, 2015: 1-4

[3] Kochte M A, Sauer M, Gomez L R, et al. Specification and verification of security in reconfigurable scan networks[C] //Proceedings of the IEEE European Test Symposium. Los Alamitos: IEEE Computer Society Press, 2017: 1-6

[4] Dworak J, Crouch A, Potter J, et al. Don't forget to lock your SIB: hiding instruments using P1687[C] //Proceedings of the IEEE International Test Conference. Los Alamitos: IEEE Computer Society Press, 2013: 1-10

[5] Zygmontowicz A, Dworak J, Crouch A, et al. Making it harder to unlock an LSIB: honeytraps and misdirection in a P1687 network[C] //Proceedings of Design, Automation \& Test in Europe Conference \& Exhibition. Dresden: European Design and Automation Association, 2014: 1-6

[6] Liu H J, Agrawal V D. Securing IEEE 1687-2014 standard instrumentation access by LFSR key[C] //Proceedings of the IEEE Asian Test Symposium. Los Alamitos: IEEE Computer Society Press, 2015: 91-96

[7] Kumar K S, Satheesh N, Mahapatra A, et al. Securing IEEE 1687 standard on-chip instrumentation access using PUF[C] //Proceedings of the IEEE International Symposium on Nanoelectronic and Information Systems. Los Alamitos: IEEE Computer Society Press, 2016: 56-61

[8] Kan S, Dworak J, Dunham J G. Echeloned IJTAG data protection[C] //Proceedings of the IEEE Asian Hardware-Oriented Security and Trust. Los Alamitos: IEEE Computer Society Press, 2016:1-6

[9] Baranowski R, Kochte M A, Wunderlich H J. Securing access to reconfigurable scan networks[C] //Proceedings of Asian Test Symposium. Los Alamitos: IEEE Computer Society Press, 2013: 295-300

[10] Baranowski R, Kochte M A, Wunderlich H J. Access port protection for reconfigurable scan networks[J]. Journal of Electronic Testing, 2014, 30(6): 711-723

[11] Atteya A, Kochte M A, Sauer M, et al. Online prevention of security violations in reconfigurable scan networks[C] //Proceedings of the IEEE European Test Symposium. Los Alamitos: IEEE Computer Society Press, 2018: 1-6

[12] Baranowski R, Kochte M A, Wunderlich H J. Fine-grained access management in reconfigurable scan networks[J]. IEEE Transactions on Computer-Aided Design of Integrated Circuits and Systems, 2015, 34(6): 937-946 
[13] Kochte M A, Baranowski R, Wunderlich H J. Trustworthy reconfigurable access to on-chip infrastructure[C] //Proceedings of International Test Conference in Asia. Los Alamitos: IEEE Computer Society Press, 2017: 119-124

[14] Elnaggar R, Karri R, Chakrabarty K. Securing IJTAG against data-integrity attacks[C] //Proceedings of the 36th IEEE VLSI Test Symposium. Los Alamitos: IEEE Computer Society Press 2018: $1-6$

[15] Das A, Touba N A. A Graph theory approach towards IJTAG security via controlled scan chain isolation[C]//Proceedings of the 37th IEEE VLSI Test Symposium. Los Alamitos: IEEE Computer Society Press, 2019: 1-6

[16] Raiola P, Kochte M A, Atteya A, et al. Detecting and resolving security violations in reconfigurable scan networks[C] //Proceedings of the 24th IEEE International Symposium on On-Line Testing and Robust System Design. Los Alamitos: IEEE Computer Society, 2018: 91-96

[17] Sun Yanrui. An algorithm for generating all maximum independent sets in an undirected graph[J]. Journal of Chinese Computer Systems, 2013, 34(8): 1862-1865(in Chinese)

(孙艳芯. 一个求无向图所有极大独立集的算法 $[\mathrm{J}]$. 小型微 型计算机系统, 2013, 34(8): 1862-1865)

[18] Marinissen E J, Iyengar V, Chakrabarty K. A set of benchmarks for modular testing of SOCs[C] //Proceedings of International Test Conference. Los Alamitos: IEEE Computer Society Press, 2002: 519-528 